\title{
Application of Artificial Dermal Graft Combined with Fascia Sleeve Flap in the Treatment of Chronic Bone and Steel Plate Exposed Wounds of Lower Extremities
}

\author{
Yong Li \\ Bengbu Medical College \\ Zhi-bo Zhang \\ Bengbu Medical College \\ Ji-song Liu (D 406084878@qq.com) \\ Bengbu Medical College \\ Zhu-min Wu \\ Bengbu Medical College \\ Xin-cheng Sun \\ Bengbu Medical College \\ Yu-tin Zhao \\ Bengbu Medical College \\ Xiang-zhou Zhang \\ Bengbu Medical College
}

Research article

Keywords: Bone exposure, Chronic wound, Plate exposure,Artificial dermis,flap

Posted Date: October 13th, 2021

DOI: https://doi.org/10.21203/rs.3.rs-942317/v1

License: (c) (1) This work is licensed under a Creative Commons Attribution 4.0 International License. Read Full License 


\section{Abstract}

Background:Bone tendon or graft exposure such as steel plate is common after severe trauma of lower extremity.

The traditional repair method is to use a variety of skin flaps to cover the exposure, but the wound can not heal after operation, or the wound dehiscence, ulcer, sinus, etc. occur again after short healing, and the bone plate is exposed again. The reason for this result is that the space around the bone plate is not well closed when the flap is covered『dead space is formed, blood and exudate accumulate, hematoma forms or infection occurs, and finally the wound breaks.Also due to swelling and contracture after flap operation,the tension of the suture between the flap and the receiving area is too large and becomes thin and cracked, forming an exposed state.In order to solve the above problems, we have carried out the research on "Application of artificial gradient combined with fascia sleep flap in the treatment of chronic bone and steel plate exposed woods of lower extremes".

Methods: In this paper, 11 cases of chronic wounds with bone exposure and skin necrosis after steel plate implantation were selected. First stage is the wound bed preparation including primary wound expansion,removal of necrotic tissue and incision of sinus wall, removal of deep necrotic bone and fibrotic scarred skin on the outer wall of steel plate to normal tissue on the outer edge of the wound, removal of precipitated peptone and purulent fur in the hole, periphery and bone space of the steel plate, and removal of tendon tissue with basal necrosis and disintegration of the wound. After vacuum sealing drainage (VSD) 1 2 weeks, the peritraumatic basal granulation tissue grew well and there was no necrotic tissue in the wound. In the second stage, the exposed bone was covered with artificial dermis, the steel plate hole or the periphery and the basal space were filled, and the exposed steel plate was completely embedded, and then the fascia sleeve flap was transplanted to cover the wound. The sural neurovascular flap was performed in 6 cases and the lateral superior malleolar artery perforator flap in 1 case.

Results:The flap survived well in all 11 cases. During the follow-up of 6 months to the removal of the plate, there was no case of rupture, exposure and sinus formation.

Conclusions:Artificial dermal covering combined with fascial sleeve flap transplantation can effectively avoid wound dehiscence or sinus formation caused by foreign body retention, infection and flap contracture,lt has good effect in repairing chronic wounds with bone plate exposure after severe trauma of lower limbs,.

\section{Background}

Lower limb injuries are mostly violent high-energySuch as crush injury, electric burn, impact injury, etc, often accompanied by bone exposuresevere fractures and tendon injuries. In some cases after orthopedic plate internal fixation, the bones[joints】tendons and plates are often exposed or sinus tract is formed, resulting from ischemia necrosis due to local skin and soft tissue swelling, or due to osteomyelitis, wound 
infection and other reasons ${ }^{[1]-[5]}$. After a period of so-called observation and treatment, the wound failed to heal, and then turned to chronic wound.In clinical practice, we often use various flaps to repair this kind of wound, but it is still common that the exposure of the bones[joints and plates or the formation of sinus tract recur ${ }^{[5]-[6]}$. In this study, after debridement and vacuum sealing draina(VSD )for wound bed preparation, artificial dermis(PELNAC,GUNZE LIMITED,JAPAN.) was used to cover exposed bone, embed exposed steel plate, fill the peripheral space of steel plate, and then fascia sleeve flap transplantation was performed, In consequence, the study produced good results.

\section{Data And Methods}

\subsection{General information $\rrbracket$}

A total of 11 cases, 9 males and 2 female, aged from 18-55 years old, There were 7 cases of traffic accident rolling, 3 cases of high-voltage electric injury and 1 case of falling from heightand. The wounds were located in the lower part of the tibia, bone exposure with sinus formation in 3 cases,including bone and steel plate exposure in 6 cases, steel plate exposure and chronic sinus in 2 cases. The time from injury to admission was 1-6 months.

\subsection{Cases selection}

\subsubsection{Inclusion criteria:}

chronic wounds with skin and soft tissue defects of lower extremities and exposure of bone and steel plate, the blood vessels of lower limbs were unobstructed, there are enough donor sites of skin flap at the back of the leg, and all patients are in good condition and can tolerate the operation and anesthesia.

\subsubsection{Excluded cases:}

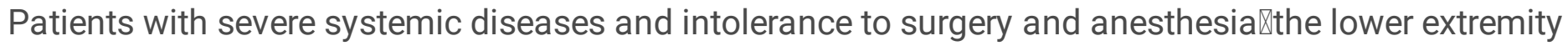
vascular injury was serious and there was no sufficient donor site,there are obvious concerns about the operation method,severe wound infection, osteomyelitis patients.

\subsection{Method CTA (Computed Tomography Angiography ) or CDFI( color doplor flow image)}

were used to check the patency of lower extremity artery and perforator before operation $\triangle \mathrm{Figure} 1 \mathrm{~A} \square$ B).Then the selected cases were operated as follows. 


\subsubsection{Wound bed preparation in first stage}

After admission, the relevant examination was perfected, and the wound was subjected to bacterial culture and drug sensitivity test. In the first stage, the wound expansion was performed under general anesthesia or combined spinal-epidural anesthesia , removing the aging granulation tissue and fibrotic scared skin around the wound, removing the scared skin from the periphery of the steel plate or the anterior wall of the sinus to healthy soft tissue, the wound was fully opened and the sinus or subcutaneous cavities were eliminated. Removing the tendinous tissue such as necrotic tendons and ligaments around the joint of the wound base, and scrape off the granulation of the wound base until there is active bleeding. Careful removing the peptone in the steel plate hole and the steps and gaps formed between the edge of the steel plate and the bone surface, and then removing the peptone in the gap between the broken end of the fracture. After cleaning the wound again, the wound was externally applied with gauze impregnated with iodine voltfor 15 minutes, observing that there was no obvious bleeding, the wound was treated with VSD immediately or 24 hours later. After 1-2 clinical periods of VSD till to obvious granulation development in the base of the wound, the second stage operation was performed.(Figure2A,B,C)

\subsubsection{The exposed joints and plates were covered with artificial dermis and grafted with fascial sleeve flap in stage II.}

After successful anesthesia, clean the wound again, the denatured and necrotic bone cortex was chiseled off $1 \mathrm{~mm}$ to $2 \mathrm{~mm}$, the peptone in the step and gap formed between the edge of the steel plate and the bone surface and the peptone in the gap between the broken end of the fracture were removed.

هFigure3:A,B\

The skin around the wound was lifted about $1 \mathrm{~cm}$ from the superficial layer of the deep fascia to the periphery to form a cuff. If the skin around the wound is scarred or severely adhered to the base, the epidermis of the wound margin can also be cut off about $1 \mathrm{~cm}$ width to form a fresh baseखFigure $4 A, B$ ), and after the exact hemostasis, the wound is washed with $75 \%$ alcohol, and the skin flap is designed according to the size of the wound. The flap was $1 \mathrm{~cm}$ enlarged than the designed area and in this expanded range, it is peeled off outward along the subdermis to form a fascia surface, cut along the outer edge of the fascia surface to the deep fascia layer, and lift the flap. A fascial sleeve of about $1 \mathrm{~cm}$ except the pedicle was formed in the periphery of the flap(Figure $5: A, B)$. If the cuff is not formed during debridement, there is no need to form a fascia sleeve. After the flap was lifted to cover the wound completely, the exposed bone and steel plate were covered with artificial dermis. The holes in the steel plate and the steps formed at the edge of the steel plate and the gaps between the steel plate and the bone should be filled closely in order to eliminate the dead cavity after transplantation (Figure6:A,B). During suture, a vertical incision was made every $5 \mathrm{~cm}$ at the skin edge of the vertical cuff in order to reduce tension and facilitate drainage, and the flap was transferred to the recipient area, then the fascial sleeve was buried in the cuff around the wound. One or two rubber patches were placed under the flap, 
then, interrupted suture was used in the dermal margin of the flap and the wound to close the wound. Fllowing, the flap was bandaged under proper pressure.After the operation, lie in bed strictly for two weeks, raise the operative limbs to prevent pressure on the flap and pedicle, and routinely perform blood volume replenishment, use anti-inflammatory, relieve pain, keep warm, etc. The dressings were changed for the first time on the third day after operation, and the survival of the flap was observed.Sural neurovascular flap was performed in 6 cases and lateral supramalleolar artery perforator flap in 1 case.

\section{Results}

All the flaps survived well in 11 cases, without marginal necrosis and poor blood supply of the flap. During a follow-up for 6 months or till to the steel plate was removed, there was no bone plate exposure or sinus formation.

\section{Typical Cases}

A female patient with right tibiofibular fracture was treated with internal fixation with steel plate. After the operation , the skin was necrotic and ruptured, resulting in the exposure of steel plate. After debridement , artificial dermis covered exposed steel plate, sural neurovascular fascia sleeve flap transplantation, the flap survived well, followed up for 6 months, there was no rupture and sinus formationखFigure7:A-L】.

\section{Discussion}

The early stage of lower limb trauma is mostly treated by orthopaedics or emergency surgery ${ }^{[7]-[10]}$.Some patients have primary injuries such as such as electric burn, severe rolling of skin and soft tissue, vascular injury, or local hematoma , fat liquefaction and hematoma infection $₫$ which eventually develop into skin necrosis ${ }^{[11]-[13]}$; For patients with fracture, plate internal fixation is a more common method. However, a lot of inflammatory exudate accumulate in the wound in some patients because of postoperative skin tissue swelling, excessive shear tension, skin necrosis or incision dehiscence or secondary wound infection, osteomyelitis.

In addition, due to the retention of foreign bodies such as dead bone, necrotic and degenerative tendon tissue and steel plate, the ejection reaction of the body also produces inflammatory exudation, which contains the colloidal substances such as proteins in the exudate precipitate and solidify in the foreign body surface and the surrounding spaces to form peptone which becomes a good culture medium for bacteria.Bacteria multiply in large numbers in the above-mentioned areas(Figure 2), release toxins, and further aggravate the inflammatory reaction of the tissue around the wound, so that the wound continues to produce exudate and pus, forming a vicious circle, and the wound can not heal ${ }^{[14]-[15]}$. After timely 
wound dressing change and anti-infective treatment, the acute inflammation of the wound was controlled and turned to chronic wound, the bone plate was exposed or formed one or more sinuses. After several months or even longer treatment, there was no obviously tending to heal.

For this kind of wounds, the traditional method is to use various flaps to repair them, but some patients split again across the position of the steel plate at the suture of the flap edge and the recipient area, and the steel plate is exposed again, certainly some flaps heal well in early time, however, 1 or 2 months after operation, the sinus tract on the suture line between the flap and the recipient area appeared again, with intermittent discharge of secretions, and the sinus orifice showed typical chronic inflammation ${ }^{[16]}$.The early swelling of the transplanted flap is an important reason for the re split of the flap and the exposure of bone steel nails, In the later stage, due to the contracture of the flap tissue and the tissue around the wound, the tension between the flap and the wound suture was too large,Because the suture between the flap and the recipient tissue is a single end-to-end contact, under the action of tension, the tissue at the suture becomes thinner and finally splits, In particular, if the suture crosses the bone or steel plate, there is no active tissue in the deep layer to provide healing and implantation, and it is only a matter of time before the wound splits again.

Some scholars reported that if the wound fails to heal in 4 weeks, it will become a chronic wound. The important characteristic of chronic wound is the stagnation of wound healing process. Once a chronic wound is formed, the tissue repair process stops, the tissue around the wound becomes fibrotic, scarred, and stops growing,The exposed bone, tendon or steel plate can not be effectively covered by tissue, resulting in lasting exposure.

Another important reason for healing failure is the residual foreign bodies in the wound and the formation of dead cavity, especially infectious foreign bodies. Necrotic bone and tendon, the steel plate as a foreign body in the wound can neither be completely removed nor effectively covered.Tissue rejection and bacterial colonization make the wound inflammatory reaction continue, and the dead cavity at the bone plate or broken end of the fracture becomes the best place for the accumulation of inflammatory exudate and pus. The protein components in the exudate solidify into a good bacterial culture medium, and the bacteria multiply to produce pus, which further stimulates the surrounding tissues to produce inflammatory reaction and form a vicious circle.A large amount of pus accumulates and disperses to the deep along the steel plate or bone to form a pus cavity. The external tissues become brittle and thin due to inflammatory reaction, and finally the skin breaks and the pus is discharged to form cracks or sinuses (Fig. 8: A, B, C).

Due to the stimulation of chronic inflammation, the tissue around the wound gradually fibrosis, scarring, congestion and blood stasis, thickening, dark purple color, lack of elasticity, poor circulation, which is not conducive to wound repair ${ }^{[17]-[22]}$.

In this study, according to the causes of the formation of secondary chronic bone exposure wound, through one-stage expansion, remove the scar skin within a certain range around the wound, cut or 
remove the sinus wall, scrape out the basal granulation tissue, and cultivate a fresh wound bed in combination with VSD,turn the chronic wound into an acute wound,reactivate the wound healing process, and provides a good basis for flap healing and wound final healing.After the preparation of the wound bed, the second stage debridement. The necrotic bone, periosteum, tendon tissue and peptone on the surface of the plate and bone were removed, the space around the bone of the plate was filled with artificial dermis, and the exposed bone and plate were completely covered.Then the wound was closed with flap transfer.Artificial dermis isolated the contact between body tissue and foreign body in the early

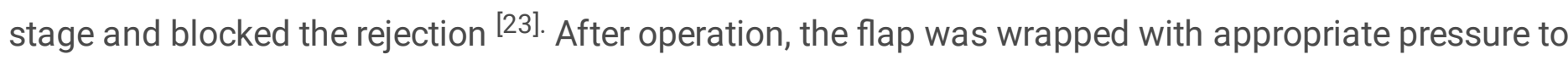
effectively prevent the possibility of dead cavity residue and blood accumulation under the flap to form pus cavity.Using fascial cuff flap transplantation, the suture between the flap edge and the wound edge tissue was changed from end-to-end plane contact to three-dimensional mortise and tenon contact, This three-dimensional contact and wound bed with rich basal blood supply greatly increases the contact area between the flap and the tissue around the wound, so that the flap and the wound can establish blood supply in an earlier and wider range, form a complete fibrous capsule earlier, and completely embed the dead bone and steel plate.At the same time, the three-dimensional mortise and tenon contact can effectively prevent the risk of skin thinning and cracking at the suture due to the excessive tension caused by the retraction of the flap.Since the bacteria in chronic wounds are mainly low toxic strains [24], after twice debridement and cleaning with VSD and alcohol, the bacterial content is greatly reduced. Fresh wound granulation tissue and flap tissue with reliable blood supply can completely remove the residual bacteria, and the possibility of infection and sinus formation has been significantly reduced.

In recent years, our unit has treated many patients with the above condition. On admission, most of the wounds were partial steel plate exposure with sinus formation, a few was all steel plate exposure, local bone exposure, bone cortex was gray yellow or gray black, with necrotic tendinous tissue exposure. In all cases, the skin around the wound was dark purple, hard and inactive. The growth of wound epidermis was reversed, and there was a small amount of purulent secretion in the wound.

When expanding the wound, the scar skin and fibrotic tissue of the outer wall of the steel plate should be excised. If there is a sinus, the outer wall of the sinus should be incision, and the fibrous capsule inside the outer wall and the sinus cavity should be removed ${ }^{[10]}$. If necessary, make a transverse incision at the bottom of the sinus wall, so that the sinus wall can be loosely attached to the base after the fibrous capsule is removed. If the fibrotic scar of the outer wall of the sinus is so severe that it can not be well attached to the base, it can be removed as appropriate. Free bone fragments should be removed. The necrotic periosteum and tendinous tissue at the base of the wound were also removed as clean as possible. Scratching the aged granulation at the bottom can cause bleeding in the wound, which can lead to fresh wounds, thus activating the wound healing process. The protein-like tissue attached and precipitated in the holes of the steel plate, in the steps formed between the edge of the steel plate and the bone surface, in the gap formed by the incompact adhesion between the steel plate and the bone, and in the gap at the broken end of the bone should be carefully cleaned to remove. During the skin flap transplantation, the denatured and necrotic bone cortex should be chiseled off $1-2 \mathrm{~mm}$, the peptone in the 
steel plate steps and spaces should be removed, and the exposed bone or steel plate should be covered with artificial dermis. The gap between the steps formed at the edge of the steel plate and the bones under the plate should be closely filled, so that the dead space will not remain after the flap transplantation and avoid infection and necrosis caused by effusion under the flap after operation.

Artificial dermis was developed by yannas and Burke in 1980. It is a membrane artificial skin and a synthetic tissue used to repair skin tissue ${ }^{[25,26]}$.S. Suzuki ${ }^{[27]}$ researched and improved the product and developed a new artificial dermis (PELNAC), which is widely used in scar shrinkage repair, excision of wounds caused by tumors, traumatic skin defects, peeling of skin flaps and other reasons,the effect evaluation shows that the good rate is $86 \%$. In recent years,PELNAC has been widely used in some wounds with bone exposure after trauma. When the artificial dermis was transplanted to the wound with poor basal blood supply, the time of local vascularization was delayed than that of the wound with good basal blood supply ${ }^{[28]}$.In this study, two debridement and VSD were performed to complete the wound bed preparation, so as to form a wide base with good blood supply around the wound, so as to ensure the vascularization of artificial dermis and the healing of flap. The blood supply of the flap is reliable. When the flap covers the artificial dermis, in a short time, the deep layer of the artificial dermis is filled with new collagen, and the sponge structure is replaced by true skin like tissue, so as to quickly complete the embedding process of steel plate or exposed bone.

\section{Abbreviations}

VSD:vacuum sealingdrainage,CTA:Computed Tomography Angiography,CDFl:color plor flow image.

\section{Declarations}

\section{Ethics approval and consent to participate:}

This study was approved by the ethics and ethics committee,all patients agreed to participate in the study.

\section{Consent for publication:}

We declare that the manuscript, including relevant data, figures and tables, has not been published before and has not been considered elsewhere. All authors agree that this manuscript will be published in Journal of Orthopaedic Surgery and Research.

\section{Availability of data and materials:}

The author promises that the data used in this paper are true and accurate. 


\section{Competing interests:}

We declare that we have no financial and personal relationships with other people or organizations that can inappropriately influence our work, there is no professional or other personal interest of any nature or kind in any product, service and/or company that could be construed as influencing the position presented in, or the review of, the manuscript entitled "Application of artificial dermal graft combined with fascia sleeve flap in the treatment of chronic bone and steel plate exposed wounds of lower extremities".

\section{Funding:}

This study was supported by Bengbu science and Technology Bureau (20200324).

\section{Authors' contributions:}

We certify that all authors have made substantial contributions to all of the following:the conception and design of the study, or acquisition of data, or analysis and interpretation of data.drafting the article or revising it critically for important intellectual content.

Acknowledgements:We thank Bengbu science and Technology Bureau for its support,and their hard work of all the authors for the development of this study. We would like to express our gratitude to the editor for his attention to this article.

\section{References}

1. Woussen A, Feuvrier D, Woussen É, Fageot J, Sergent P, Leclerc G, Obert L, Pluvy I. Extensor digitorum brevis muscle flap for lower extremity coverage in a context of posttraumatic sepsis. Orthop Traumatol Surg Res. 2021 Mar 22:102901. doi: 10.1016/j.otsr.2021.102901. Epub ahead of print. PMID: 33766678.

2. Khan AM, Tang QO, Spicer D. The epidemiology of adult distal femoral shaft fractures in a central Iondon major trauma centre over five years. Open Orthop J. 2017;11:1277-91.

3. Meng WZ, Guo YJ, Liu ZK, Li YF, Wang GZ. [Influencing factors for trauma-induced tibial infection in underground coal mine]. Zhonghua Lao Dong Wei Sheng Zhi Ye Bing Za Zhi. 2016 Jul 20;34(7):523525. Chinese. doi: 10.3760/cma.j.issn.1001-9391.2016.07.011. PMID: 27682489.

4. Lou X, Xue H, Li G, Wang K, Zhou P, Li B, Chen J. One-stage Pelnac Reconstruction in Full-thickness Skin Defects with Bone or Tendon Exposure. Plast Reconstr Surg Glob Open. 2018 Mar 21;6(3):e1709. doi: 10.1097/GOX.0000000000001709. PMID: 29707464; PMCID: PMC5908513.

5. Woussen A, Feuvrier D, Woussen É,Fageot J, Sergent P, Leclerc G,Obert L,Pluvy I.Extensor digitorum brevis muscle flap for lower extremity coverage in a context of posttraumatic sepsis. Orthop 
Traumatol Surg Res. 2021 Mar 22:102901. doi: 10.1016/j.otsr.2021.102901. Epub ahead of print. PMID: 33766678.

6. Varey AHR, Khan U. Soft-tissue reconstruction of open fractures of the lower limb: muscle versus fasciocutaneous flflaps. Plast Reconstr Surg. 2013;131:448e.

7. Gierer P, Wichelhaus A, Rotter R. Die Olekranonfraktur [Fractures of the olecranon]. Oper Orthop Traumatol. 2017 Apr;29(2):107-114. German. doi: 10.1007/s00064-017-0490-z. Epub 2017 Mar 16. PMID: 28303286.

8. Buza JA 3rd, Leucht P. Fractures of the talus: Current concepts and new developments. Foot Ankle Surg. 2018 Aug;24(4):282-290. doi: 10.1016/j.fas.2017.04.008. Epub 2017 Apr 24. PMID: 29409210.

9. Kancherla VK, Singh A, Anakwenze OA. Management of Acute Proximal Humeral Fractures. J Am Acad Orthop Surg. 2017 Jan;25(1):42-52. doi: 10.5435/JAAOS-D-15-00240. PMID: 28002214.

10. Boopalan PR, Nithyananth M, Titus VT, Cherian VM, Jepegnanam TS. Experience of using local flaps to cover open lower limb injuries at an Indian trauma center. J Emerg Trauma Shock. 2011 Jul;4(3):325-9. doi:10.4103/0974-2700.83806. PMID: 21887018; PMCID: PMC3162697.

11. Riechelmann F, Kaiser $P$, Arora R. Primäres Weichteilmanagement bei offenen Frakturen [Primary soft tissue management in open fracture]. Oper Orthop Traumatol. 2018 Oct;30(5):294-308. German. doi: 10.1007/s00064-018-0562-8. Epub 2018 Sep 4. PMID: 30182178.

12. Zalavras CG. Prevention of infection in open fractures. InfectDis ClinNorth Am. 2017;31(2):339-52.

13. Wang J, Wu Y, Wang Y, Wang M. [Conservative dressing change method in treatment of skin necrosis after open reduction with internal fixation of calcaneal fracture]. Zhongguo Xiu Fu Chong Jian Wai Ke Za Zhi. 2011 Jul;25(7):805-7. Chinese. PMID: 21818944.

14. Groupe de pathologie infectieuse. Recommandations de pratiques cliniques. Infections osteoarticulaires sur matériel (prothese, implant, ostéosynthese). Med Mal Infect 2009.

15. Gupta P, Singh HS, Shukla VK, Nath G, Bhartiya SK. Bacteriophage Therapy of Chronic Nonhealing Wound: Clinical Study. Int J Low Extrem Wounds. 2019 Jun;18(2):171-5. doi:10.1177/1534734619835115. Epub 2019 May 13. PMID: 31081402.

16. Xiaohong Z, Minggang W, Qingfu S, Yanjun C, Tao L. [Reconstruction of tibial exposure with local muscular flap, VSD and skin transplantation]. Zhonghua Zheng Xing Wai Ke Za Zhi. 2015 Jul;31(4):277-80. Chinese. PMID: 26665928.

17. Edwards R, Harding KG. Bacteria and wound healing. Curr Opin Infect Dis. 2004 Apr;17(2):91-6. doi: 10.1097/00001432-200404000-00004. PMID: 15021046.

18. Gjødsbøl K, Christensen JJ, Karlsmark T, et al. Multiple bacterial species reside in chronic wounds: a longitudinal study. Int Wound J. 2006;3(3):225-31.

19. Wang $T$, Feng $Y$, Sun $H$, et al. miR-21 regulates skin wound healing by targeting multiple aspects of the healing process. Am J Pathol. 2012;181(6):1911-20. 
20. Serra R, Grande R, Buffone G, et al. Extracellular matrix assessment of infected chronic venous leg ulcers: role of metalloproteinases and inflammatory cytokines. Int Wound J 2014. [Epub ahead of print.

21. Serra R, Buffone G, Costanzo G, et al. Altered Metalloproteinase-9 expression as the least common denominator between Varicocele, Inguinal Hernia and Chronic Venous Disorders. Ann Vasc Surg. 2014;28(3):705-9.

22. Serra R, Gallelli L, Conti A, et al. The effects of Sulodexide on both clinical and molecular parameters in patients with mixed arterial and ulcers of lower limbs. Drug Des Devel Ther. 2014;8:1-9.

23. Jiang $D$, Chen $B, X u M, H u$ D, Tang C, Zhu X. [The manufacturing and clinical application of heterogenous acellular dermal matrix]. Zhonghua Shao Shang Za Zhi. 2002 Feb;18(1):15-8. Chinese. PMID: 12515660.

24. Serra R, Grande R, Butrico L, Rossi A, Settimio UF, Caroleo B, Amato B, Gallelli L, de Franciscis S. Chronic wound infections: the role of Pseudomonas aeruginosa and Staphylococcus aureus. Expert Rev Anti Infect Ther. 2015 May;13(5):605-13. doi: 10.1586/14787210.2015.1023291. Epub 2015 Mar 8. PMID: 25746414.

25. [2]Yannas IV, Burke JF. Design of an artificial skin.I.Basic design principles[J]. J Biomed Mater Res. 1980;14(1):65-81.

26. Burke JF, Yannas IV, Jr QW,et al.Successful use of a physiologically acceptable artificial skin in the treatment of extensive burn injury[J]. Annals of Surgery,1981,194(4):413-28.

27. Suzuki S, Kawai K, Ashoori F,et al. Long-term follow-up study of artificial dermis composed of outer silicone layer and inner collagen sponge[J]. Br J Plast Surg. 2000;53(8):659-66.

28. Koga Y, Komuro Y, Yamato M,et al.Recovery course of full-thickness skin defects with exposed bone:an evaluation by a quantitative examination of new blood vessels[J].The Journal of surgical research,2007,137(1):30.

\section{Figures}



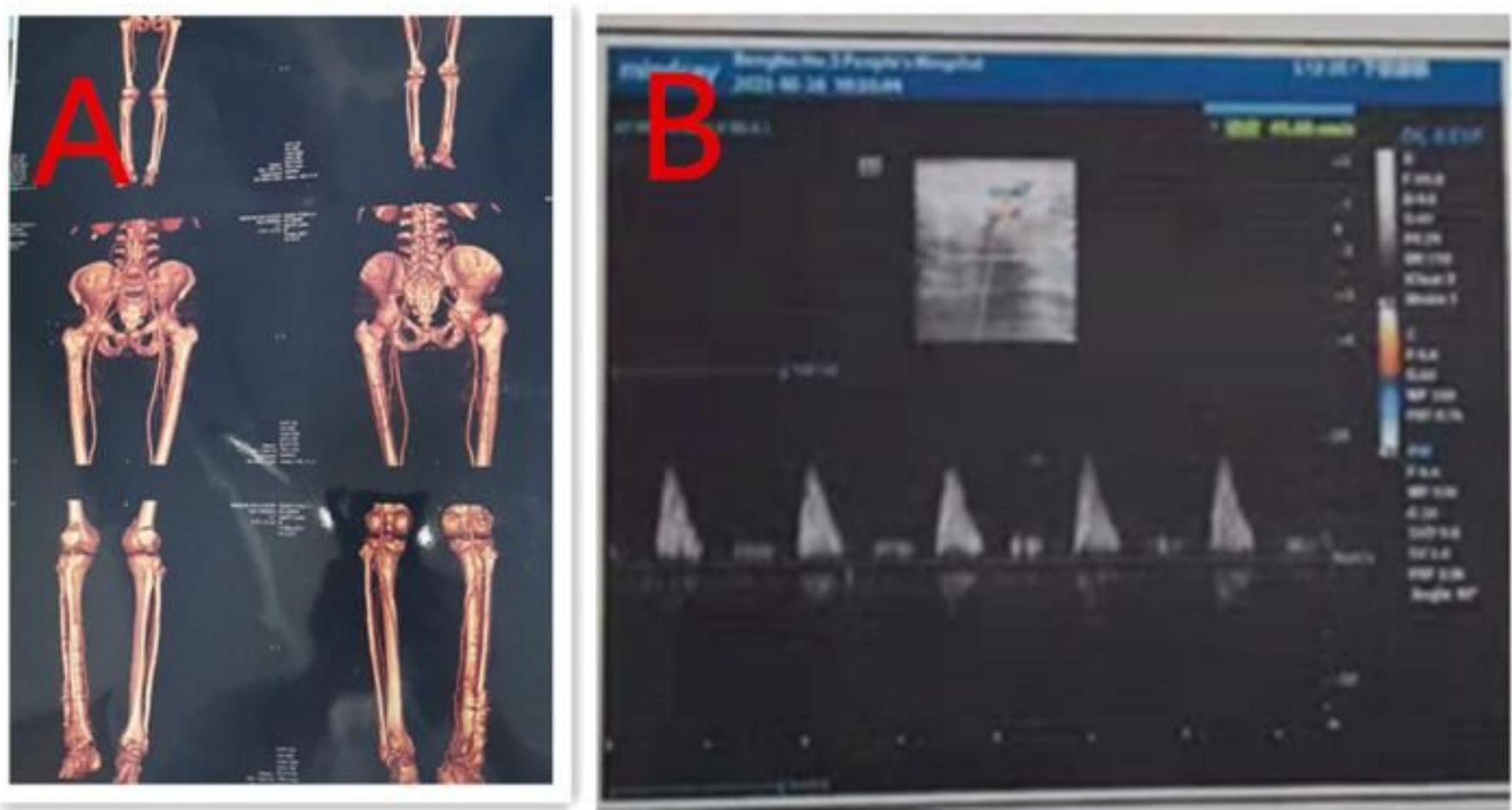

\section{Figure 1}

$\mathrm{CTA}(\mathrm{A})$ or $\mathrm{CDFI}(\mathrm{B})$ were used to check the patency of blood vessels before operation. 



\section{Figure 2}

$A \bowtie$ Preoperative,B: After the first stage of debridement,C:VSD


Figure 3

$A, B$ : Peptone in the gap of steel plate 

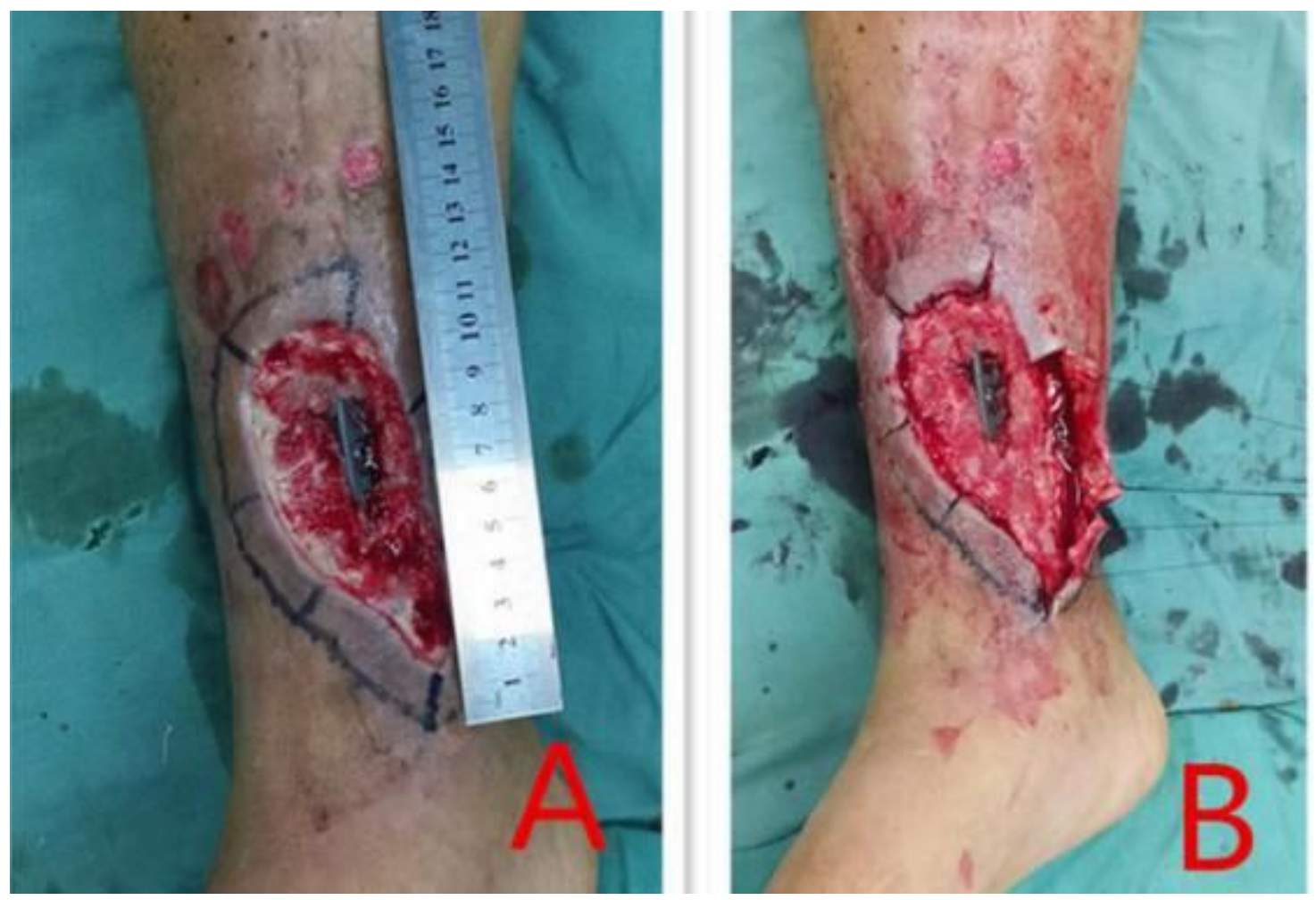

Figure 4

$\mathrm{A} \otimes S$ leeve design $₫ \mathrm{~B} \otimes$ Lift the sleeve
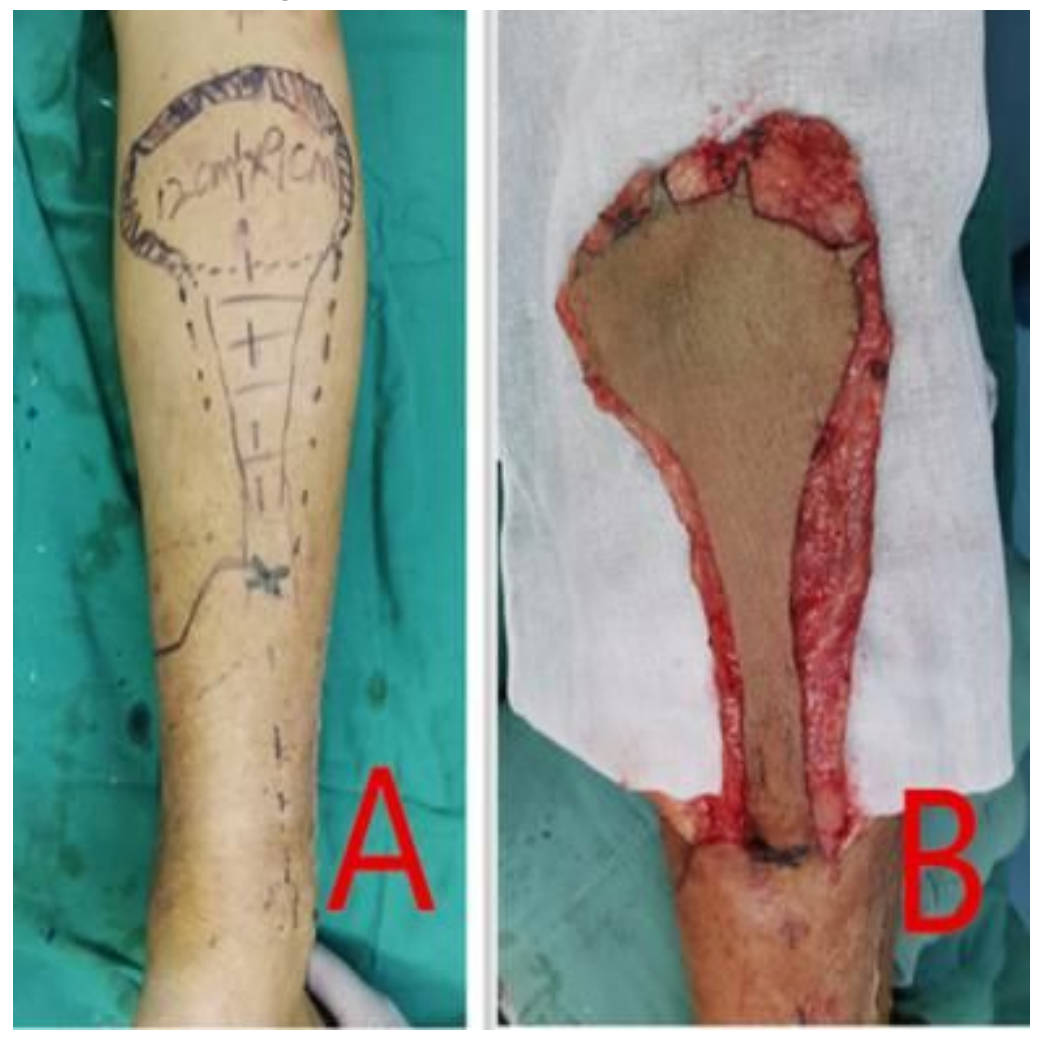

Figure 5

A:Design of fascial sleeve flap B囚Fascial sleeve flap 

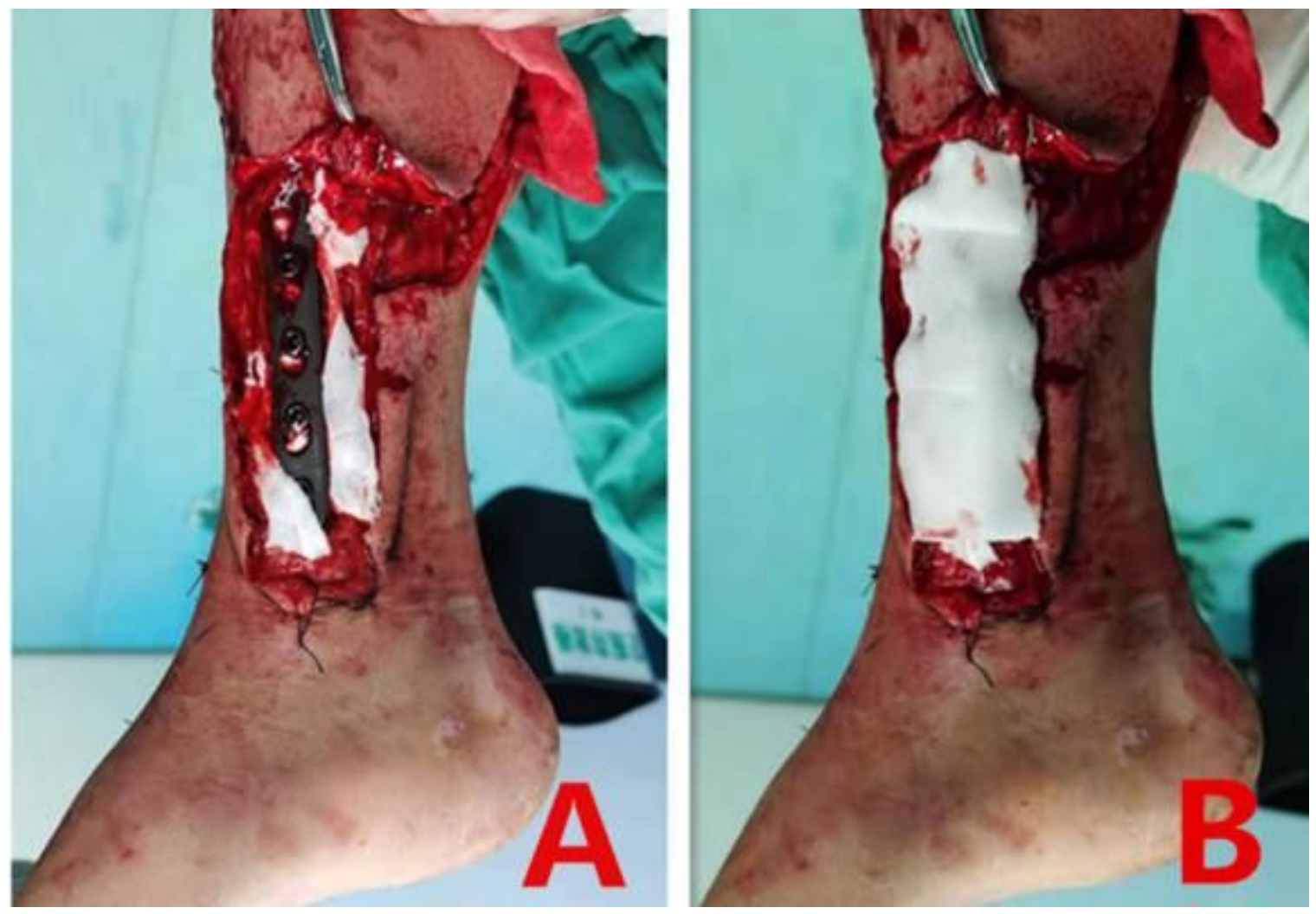

Figure 6

A: Filling the pores and gaps of steel plate with artificial dermis, B:Cover the exposed steel plate with artificial leather 

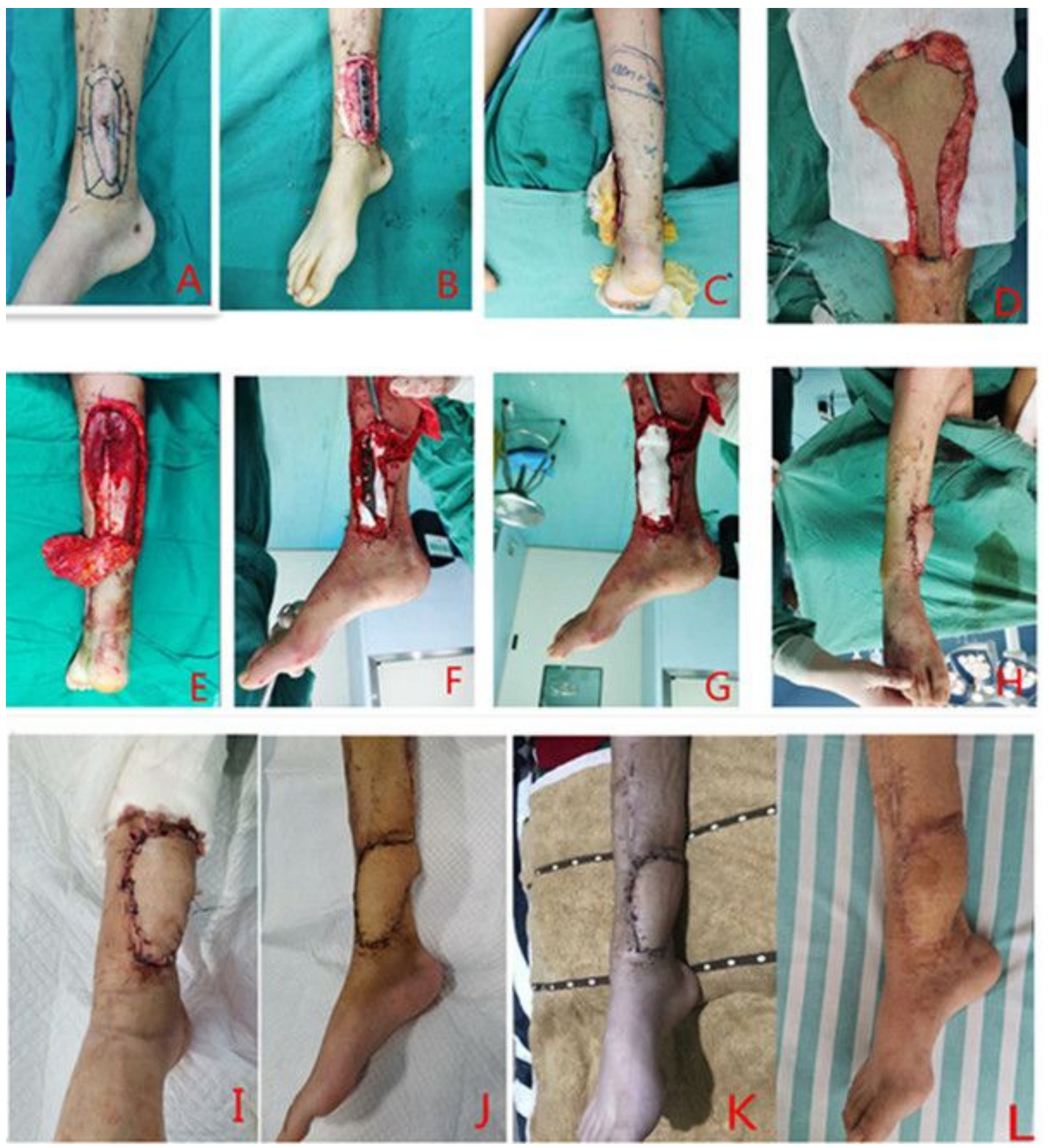

Figure 7

A:Preoperative, debridement and incision design,B:Expand the wound, lift the sleeve skin, and clean the peptone in the gap between the steel plates,C:Design of sural nerve fascial sleeve flap,D:Fascial sleeve flap,E:Flap lifting,F:Filling steel plate pores with artificial dermis,G:Artificial dermis embedded steel plate,H:Immediate treatment of flap transfer,l:Three days after operation,J:2 weeks after operation,K:One month after operation,L :4 months after operation. 



Figure 8

A, B, C:The sinus was formed again after flap covering 